\title{
THE DISTRIBUTION OF COLLOIDAL ARSENIOUS SULPHIDE BETWEEN THE TWO LIQUID PHASES IN THE SYSTEM WATER, ETHER, ALCOHOL
}

\section{BY HARRY P. CORLISS}

The present paper gives the results of some quantitative measurements of the distribution of colloidal arsenic trisulphide between the two liquid phases formed when ether, water and alcohol are mixed in proper proportions; that equilibrium involving the distribution of colloids may be realized experimentally was established in 1908 by Lash Miller and $\mathrm{McPherson},{ }^{1}$ who made also a preliminary study of the conditions affecting the distribution ratio.

The binodal curve and the tie lines at $o^{\circ} \mathrm{C}$ for the system Ether-water-alcohol were determined by Bonner, ${ }^{2}$ who employed a method designed rather to permit measurement with very small quantities than to furnish extremely accurate results. As a preliminary, therefore, it was necessary to redetermine the curve and lines; accurate specific gravity measurements also were made of solutions of known composition on the binodal curve, and a graph was constructed so that the composition of any one of a pair of solutions in equilibrium could be found simply by determining its density.

Satisfactory methods of preparing the colloidal solutions and of determining their content of arsenic were then worked out, and a method of allowing for the effect of the colloid on the specific gravity of the solutions was checked experimentally. Finally, some twenty measurements of the distribution ratio were made and their results collated.

${ }^{1}$ Jour. Phys. Chem., I2, 709 (1908); see also Reinders: Zeit. KolloidChemie, 13, 235 (1913).

${ }^{2}$ Jour. Phys. Chem., I4, 738 (1910); a very complete study of the system alcohol-water-ether at $25^{\circ} \mathrm{C}$ has been made by Shinkichi Horiba, see Memoirs of the College of Science and Engineering, Kyoto Imperial University, Vol 3, No. 3, p. 63 (IgII). 


\section{Binodal Curve, Tie-lines, Specific Gravities}

In determining the points of the binodal curve, and in all other experiments of this paper, Kahlbaum's ether "über Natrium destilliert," and Kahlbaum's "ethyl alcohol 99.8 percent" were used; account was taken of the water added with the alcohol; the total weight of the liquids used in each determination of a point on the binodal curve was about 25 grams.

The liquids were weighed into the mixing tube from pycnometers of the Ostwald-Sprengel type, provided with a long fine exit-tube, suitably bent. The tube in which the liquids were mixed was about ro $\mathrm{cm}$ long and $2.5 \mathrm{~cm}$ in diameter. Into the mouth was fitted tightly a cork through which passed a short glass tube of about $\mathrm{I} \mathrm{cm}$ diameter, and over the end of this tube two overlapping pieces of thin sheet rubber were drawn and tied. This allowed the end of a pycnometer to be inserted with the least loss of vapor; and while containing the liquids and immersed in ice water, no smell of alcohol or ether could be detected, showing that the sheet rubber was an effective means of closing the tube.

A point on the binodal curve was determined by blowing over from the pycnometers into the tube the desired amounts of ether and water; then adding alcohol in quantity nearly but not quite enough to make all homogeneous, the tube being all the time immersed in ice water to keep the vapor tension as low as possible. The tube was then removed to an ice bath provided with a stirrer run by a small motor, and allowed to cool for about fifteen minutes more, being frequently stirred by giving the liquid in the tube a rotating motion. Then a little more alcohol was added from the pycnometer, mixed well by rotating and gently shaking, and placed again in the ice bath to see if it would separate into layers. The final addition of alcohol was made easy, however, without waiting long for separation to occur, by the fact that even when a long time would be required for separation to take place, a silky appearance of the liquid, when shaken, was seen; and then the alcohol was added, drop by drop, until the last drop just caused this silky appearance to dis- 
appear. That this method of judging the final addition of alcohol was accurate, was tested many times; whenever a silky appearance was noted, separation always, occurred on standing in the bath, and no separation occurred after just enough alcohol had been added to dissipate this silky appearance on shaking. Of course, when points on the binodal curve where the two layers were of widely different specific gravities were being found, separation took place more rapidly than near the top of the curve, so that actual separation was obtained quickly; in such cases the diminishing amounts of either upper or lower phase served to indicate how much more alcohol should be added to make homogeneous. After the liquid was made homogeneous the specific gravity was taken. Such an experiment gave the composition and specific gravity for one point in the binodal curve.

The results of these measurements are given in Table I and Fig. I, in which the weights of ether and water taken

Table I-Experimental Data for Binodal Curve Temperature $0^{\circ} \mathrm{C}$ Specific Gravity of Water at $0^{\circ} \mathrm{C}$ r.oooo

\begin{tabular}{l|l|l|l}
\hline Ether & Water & Alcohol & Specific gravity \\
\hline 0.128 & 0.872 & 0.237 & 0.9619 \\
0.199 & 0.801 & 0.338 & 0.9397 \\
0.291 & 0.709 & 0.365 & 0.9175 \\
0.295 & 0.705 & 0.369 & 0.9160 \\
0.345 & 0.655 & 0.375 & 0.9044 \\
0.390 & 0.610 & 0.378 & 0.8939 \\
0.446 & 0.554 & 0.388 & 0.8822 \\
0.596 & 0.494 & 0.383 & 0.8698 \\
0.549 & 0.451 & 0.385 & 0.8600 \\
0.597 & 0.403 & 0.377 & 0.8500 \\
0.649 & 0.351 & 0.361 & 0.8383 \\
0.724 & 0.276 & 0.343 & 0.8227 \\
0.817 & 0.183 & 0.301 & 0.8017 \\
0.873 & 0.127 & 0.278 & 0.7878 \\
0.915 & 0.085 & 0.207 & 0.7734 \\
0.958 & 0.042 & 0.135 & 0.7575 \\
$0.488^{1}$ & 0.512 & 0.385 & 0.8738 \\
\hline
\end{tabular}

${ }^{1}$ Plait point obtained by graphic interpolation from tie-line graph and binodal curve. 


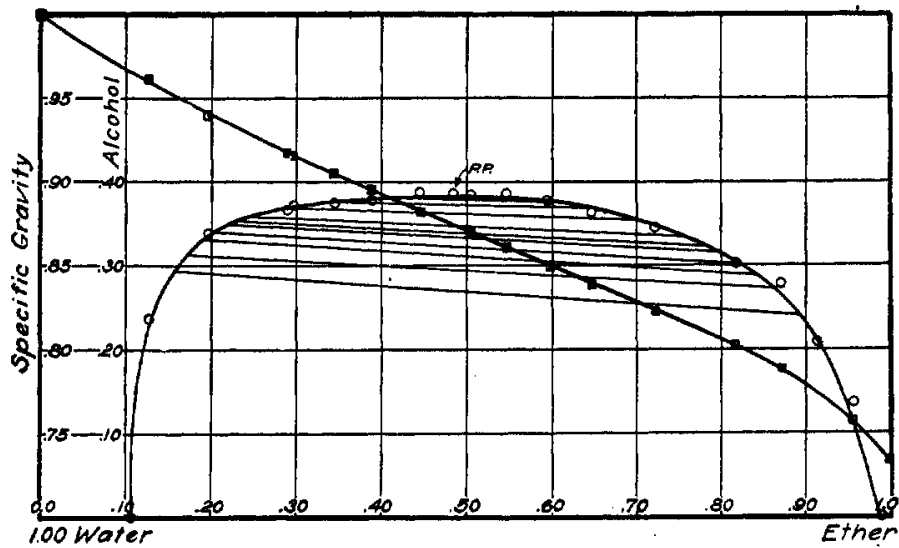

Fig. I-Binodal curve and tie-lines

together amount to one gram; the weight of alcohol given is therefore the amount needed to make one gram of the etherwater mixture homogeneous. The data of Table II were obtained by graphic interpolation from Table I; the last two

Table II-Interpolated Values for Binodal Curve

\begin{tabular}{|c|c|c|c|c|}
\hline $\begin{array}{c}\text { Ether } \\
e\end{array}$ & $\begin{array}{c}\text { Water } \\
w\end{array}$ & $\begin{array}{c}\text { Alcohol } \\
a\end{array}$ & $\log \frac{w}{a}$ & $\log \frac{e}{a}$ \\
\hline 0.980 & 0.020 & 0.035 & $\overline{\mathbf{I}} \cdot 757$ & I. 447 \\
\hline 0.975 & 0.025 & 0.060 & $\underline{\bar{x}} \cdot 620$ & I. $21 \mathrm{II}$ \\
\hline $0.95^{\circ}$ & 0.050 & 0.145 & $\overline{\mathrm{I}} \cdot 53^{8}$ & $0.8 \mathrm{I} 6$ \\
\hline 0.900 & 0.100 & 0.240 & $\overline{\mathbf{I}} .620$ & 0.574 \\
\hline 0.850 & O. I $5^{\circ}$ & 0.290 & $\overline{\mathrm{I}} \cdot 7 \mathrm{I} 4$ & 0.467 \\
\hline 0.800 & 0.200 & 0.3 I 5 & $\bar{I} .803$ & 0.405 \\
\hline 0.750 & $0.25^{\circ}$ & 0.335 & $\bar{I} \cdot 873$ & $0.35^{\circ}$ \\
\hline 0.700 & 0.300 & $0.35 \mathrm{I}$ & $\bar{I} \cdot 932$ & 0.300 \\
\hline 0.650 & $0.35^{\circ}$ & 0.365 & $\overline{\mathbf{I}} \cdot 982$ & $0.25 \mathrm{I}$ \\
\hline 0.600 & 0.400 & 0.376 & 0.027 & 0.203 \\
\hline $0.55^{\circ}$ & 0.450 & 0.384 & 0.069 & 0.156 \\
\hline 0.500 & 0.500 & $0.3^{8} 5$ & O. I I 4 & O. II 4 \\
\hline $0.45^{\circ}$ & $0.55^{\circ}$ & 0.384 & o. I 56 & 0.069 \\
\hline 0.400 & 0.600 & 0.382 & o. 196 & 0.020 \\
\hline $0.35^{\circ}$ & 0.650 & 0.377 & 0.237 & $\overline{\mathrm{I}} \cdot 968$ \\
\hline 0.300 & 0.700 & 0.37 I & 0.276 & $\overline{\mathrm{I}} \cdot 908$ \\
\hline 0.250 & 0.750 & 0.360 & 0.319 & $\overline{\mathrm{I}} .842$ \\
\hline 0.200 & 0.800 & 0.339 & 0.373 & $\overline{\mathrm{I}} \cdot 77 \mathrm{I}$ \\
\hline 0.150 & 0.850 & $0.28 \mathrm{I}$ & 0.481 & $\bar{I} \cdot 727$ \\
\hline
\end{tabular}


columns give the "logarithmic coördinates" of Bancroft's formula, ${ }^{1}$ plotted in Fig. II.

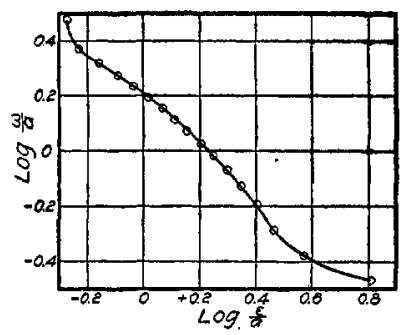

Fig. II-Binodal curve (logarithmic coördinates)

Specific Gravities.-For the purpose of taking specific gravities the left-hand pan-rest was removed from a Sartorius analytical balance which had pan-rests that rose through a hole in the base. To the bottom of the pan a hook was cemented and from it hung a platinum wire extending through the base and shelf on which the balance sat. Below the balance shelf a cork of the size to fit the mixing tube was rigidly clamped, and through it a small glass tube was fitted. From the platinum wire a human hair extended through the glass tube in the cork to a distance below sufficient to have the bulb, whose loss in weight was measured, hang immersed in the liquid when the tube was placed on the stopper. The bulb was made of glass of about $4 \mathrm{cc}$ volume, partially filled with mercury, and was suspended by a small platinum hook fastened to the end of the hair. The hair suspension proved very satisfactory; fine tungsten wire was also tried. In making a specific gravity determination, the tube containing the liquid was removed from the ice bath and placed quickly on the stopper below the balance; the ice bath was then raised, so that the liquid would be kept at $0^{\circ} \mathrm{C}$ in the new position. After a few minutes, ${ }^{2}$ to allow the liquid to cool completely again, the weight of the bulb, immersed in the liquid, was taken. From the loss in weight in the liquid and the loss in

${ }^{1}$ See Bonner: Jour. Phys. Chem., 14, 738 (I910).

2 The mixing tube and its contents left in the ice bath, with frequent stirring, fell from $18^{\circ} \mathrm{C}$ to $\mathrm{O} .1^{\circ} \mathrm{C}$ in five minutes, 
water, the specific gravity was calculated; the results of the measurements are given in Table I and Fig. I.

Tie-lines.-For the determination of the tie-lines, ether water and alcohol were mixed in the proportion to give a tieline at the approximate position desired, about Ioo grams in all being taken for each experiment. This mixture was allowed to cool and separate into layers in the ice bath; a portion of each layer was siphoned off by a glass tube and its specific gravity taken; and, from the specific gravity and binodal curves, the composition of the phases at the end of the tie-line was determined. Since the loss of weight in water was about four grams for the specific gravity bulb used, and this loss could be determined within $0.2 \mathrm{mg}$, the specific gravities could easily be determined to four decimal places; the composition of the solutions of the binodal curve, of course, are not known with any such accuracy. The results are given in Table III and in Fig. III. The composition of the solution at the plait point was ascertained by prolonging the curve of Fig. III, and choosing the point on the extrapolated curve whose ordinate was equal to its abscissa; the result is in good agreement with that obtained by interpolation in Fig. I.

TABLE III-EXPERIMENTAL DATA FOR TIE-LINES (Specific gravities at $0^{\circ} \mathrm{C}$ referred to water at $0^{\circ} \mathrm{C}$ as $\mathrm{r} .0000$ )

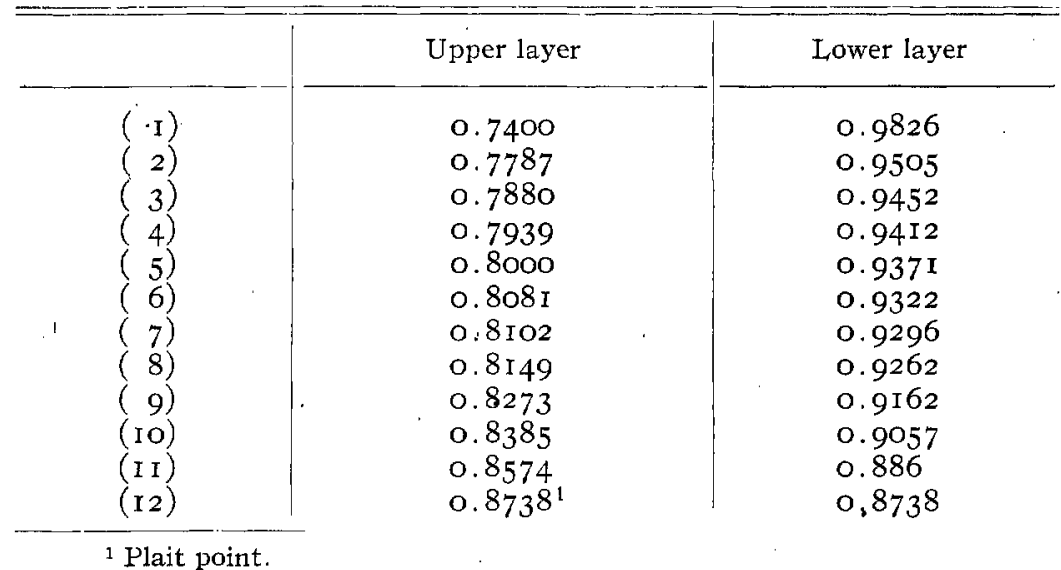




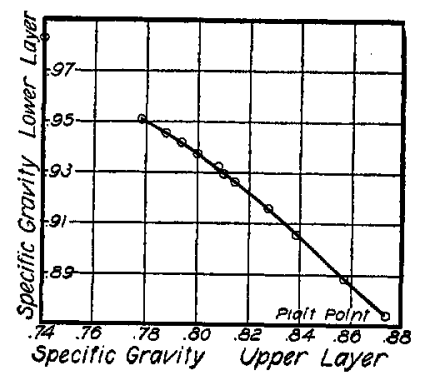

Fig. III-Tie-line plot

\section{The Colloidal Solutions}

A solution of arsenious acid was prepared by boiling water to remove air, adding about one gram arsenious oxide per hundred grams of water and boiling slowly until all was dissolved. The solution was then cooled, an equal volume of alcohol was added, and hydrogen sulphide was passed in to excess. The whole was then allowed to stand some hours, usually over night, and then hydrogen ${ }^{1}$ was bubbled through until paper moistened with silver nitrate and held over the mouth of the tube was no longer blackened. Both gases were washed by passing through two wash bottles of water.

The colloidal arsenious sulphide prepared in solutions containing alcohol or ether or both was much clearer than when water alone was used; if ether was present, however, the solution on standing underwent some change, the effect of which was that, on coagulating with acid and filtering; the filtrate contained considerable amounts of arsenic. If only water and alcohol were present, the amount of arsenic present in the filtrate after coagulation was small, and was constant for a given preparation. The change in the ether solutions was probably due to oxidation induced by the ether. ${ }^{2}$

Removal of. Hydrogen Sulphide by Lead Carbonate.-If a colloidal solution of arsenious sulphide containing alcohol

1 Picton: Jour. Chem. Soc., 6I, I37 (1892),

2 Argo: Trans. Roy. Soc. Canada, I9r3, Sec. III. 
and excess hydrogen sulphide be cooled in ice water, shaken with lead carbonate, and quickly filtered, using a funnel jacketed with ice, the excess of hydrogen sulphide is removed, and after coagulation but a small amount of arsenic is found in the filtrate. Quick work is necessary, however, as on prolonged shaking with lead carbonate the liquid is completely decolorized and the filtrate after coagulation contains arsenic; if a solution containing no free hydrogen sulphide is used, the lead salt is colored salmon to brown, if hydrogen sulphide be present, black.

Coagulation.-The sulphide is not so easily coagulated in solutions containing ether and alcohol as in pure aqueous solutions. Hydrogen sulphide in excess was passed into a solution made up from $35 \mathrm{cc}$ one-percent arsenious oxide, $25 \mathrm{cc}$ alcohol, and $20 \mathrm{cc}$ ether. To $3 \mathrm{cc}$ of this were added $5 \mathrm{cc}$ alcohol and $5 \mathrm{cc}$ water, and then a measured amount of salt solution; the mixture was let stand 5 minutes and was compared in reflected light with a sample to which no salt had been added. If no turbidity was observable, the experiment was repeated, using more of the salt solution, and so on until a slight turbidity was noticed. The following results were obtained:

Turbidity produced by

$0.08 \mathrm{cc}$ alum solution, containing $0.0 \mathrm{r}$ gram-mols ${ }^{1}$ per liter

$0.40 \mathrm{cc}$ barium chloride solution, 0.01 gram-mols per liter

$0.50 \mathrm{cc}$ hydrochloric acid, o.or gram-mols per liter

Alum is thus the best coagulant of the three, and hydrochloric acid the worst, the same as in aqueous solutions; but there is not nearly as much difference as in the latter case, the ratios being $I: 5: 6.3$, while Linder and Picton ${ }^{2}$ found the ratios $1: 32: 1590$ for aqueous solutions.

Determination of Arsenic.-The arsenic was estimated in solutions containing sulphide, ether, alcohol and water, by evaporating to dryness in a beaker on a water bath, after co-

${ }^{1} \mathrm{KAl}\left(\mathrm{SO}_{4}\right)_{2}-12 \mathrm{H}_{2} \mathrm{O}$.

${ }^{2}$ Jour. Chem. Soc., 67, 65 (1895). 
agulating with a drop of sulphuric acid. About I5 $\mathrm{cc}$ of concentrated sulphuric acid was then added and the whole heated on a gauze for some time after dense white fumes began to come off, or until the solution became quite clear and all free sulphur had disappeared; the sulphur dioxide formed keeps the arsenic reduced as arsenious acid. It was then cooled, diluted, nearly neutralized with ammonia, and then made alkaline with a saturated solution of ammonium or sodium bicarbonate, using about ro cc in excess of that necessary for neutralization. The solution was then cooled in ice water and titrated with tenth or hundredth normal iodine according to the amount of arsenic present. The volume of final solution titrated was usually about $300 \mathrm{cc}$; starch was used as indicator and a small crystal of potassium iodide was added.

Determination of Hydrogen Sulphide.-In connection with this work a convenient method of determining the strength of an aqueous solution of hydrogen sulphide was worked out. A known volume of the solution was run into a measured excess of volumetric silver nitrate; a little precipitated calcium carbonate free from chlorides was then added, and the whole boiled and filtered. The excess of silver in the solution was then determined volumetrically by sodium chloride with potassium chromate as indicator. This method was found to be more reliable than oxidation with iodine.

Effect of $A s_{2} S_{3}$ on the Binodal Curve.-It seemed very improbable that the presence of the colloidal sulphide could affect the composition of the solutions of the binodal curve; but, to make sure, a direct determination was made, as described on page 682 , using in place of water an aqueous solution containing I I.7 $\mathrm{g}$ arsenious sulphide per liter, and subtracting the weight of colloid to obtain the weight of water used. The result (expressed as in Table I) was:

Ether 0.494 Water 0.506 Alcohol 0.385 Sp.gr. 0.8748 giving a point that fits right on the curve obtained in absence of arsenic. 
Effect of $\mathrm{As}_{2} \mathrm{~S}_{3}$ on the Specific Gravity.-One cubic centimeter of the solution of the preceding paragraph thus weighs 0.8748 gram and contains

$$
0.8748 \times 0.017_{7} \times 0.506 / 1.1385=0.0037 \mathrm{~g} . \mathrm{As}_{2} \mathrm{~S}_{3}
$$

The specific gravity of amorphous arsenious sulphide is $2.76 ;^{1}$ assuming that of the suspended sulphide to be the same, the volume of the 0.0037 gram would be $0.0014 \mathrm{cc}$, and the remaining $0.8748-0.0047=0.87 \mathrm{II}$ gram of liquid would occupy $\mathrm{r} .0000-0.00 \mathrm{I}_{4}=0.9866 \mathrm{cc}$, corresponding to a specific gravity of 0.8723 which agrees well with the interpolated specific gravity 0.8724 of the arsenic free solution of the binodal curve.

As a further check, a "tie-line determination" was made, the arsenic content and the specific gravity of upper and lower layers being determined. The gravities were then corrected for the arsenious sulphide present, and the "corrected" values were found to lie on the line in Fig. III. The numbers found were:

Upper layer $0.0007 \mathrm{~g} \mathrm{AS}_{2} \mathrm{~S}_{3}$ per cc, Sp. gr. $0.86 \mathrm{I} 2$ corr. 0.8608 Lower layer $0.0074 \quad A_{2} S_{3}$ per cc, Sp. gr. 0.8904 corr. 0.8855

\section{Distribution Experiments}

Measured amounts of the aqueous-alcoholic colloidal solution of arsenious trisulphide were brought into a cylindrical glass stoppered 200 cc bottle, about $\mathrm{I} 3 \mathrm{~cm}$ tall; enough alcohol, water and ether were then added to nearly fill the bottle with a two phase liquid system of the desired composition. The whole was kept in the ice bath, and when the lower layer had settled a little, leaving the upper layer transparent, the amount of sulphide in the latter could be roughly estimated by the color. Very small amounts of the reagents, sometimes but one or two drops, were then added, until after shaking and letting stand the upper layer showed the color desired.

After complete separation of the phases had taken place, about $25 \mathrm{cc}$ of the upper layer was siphoned off and its specific

${ }^{1}$ Hausmann: Liebig's Ant1., 74, I99 (1850). 
gravity taken. Two other portions of the upper and lower layers, usually $25 \mathrm{cc}$ of upper and Io $\mathrm{cc}$ of lower layer, were withdrawn for estimation of total arsenic; and still another portion of each layer was coagulated with a drop of sulphuric acid, filtered, and the arsenic in the filtrate determined. The difference between "total arsenic" and "arsenic in filtrate" gives arsenic as arsenious sulphide.

The first determinations (Colloids I and II) were made from small amounts of freshly. prepared colloidal solutions; but as they showed that different colloidal solutions may give different ratios of distribution for the same composition of the phases (as regards ether, water and alcohol) a stock of colloidal solution (Colloid III) was prepared from $400 \mathrm{cc}$ of I percent aqueous solution of arsenious oxide and an equal volume of alcohol as described on page 687 , and was used in experiments (5) to (I3); colloids IV and V were likewise made up in quantity.

The results of the measurements are given in Table IV; the composition of the upper layer is recorded by the method of Table I; in expt. ( $I$ ), for instance, for every $0.55^{2}$ gram. ether in the upper layer there was 0.448 gram water and enough alcohol to make up a solution of the binodal curve. Under $\mathrm{As}_{2} \mathrm{O}_{5}$ is given the result of the arsenic determination in the filtrate after coagulation, calculated as arsenic anhydride; and under $\mathrm{As}_{2} \mathrm{~S}_{3}$ the difference between total arsenic and arsenic as $\mathrm{As}_{2} \mathrm{O}_{5}$, calculated as $\mathrm{As}_{2} \mathrm{~S}_{3}$.

The data of Table IV are plotted in Fig. IV; the points joined by a curve were all made with one colloidal solution (Colloid III) and were made with the fewest number of additions of reagents to get distribution, and hence with least amount of shaking and in the shortest time; the average time in the ice bath being about three hours. Points $\mathbf{I}_{2}$ and $\mathrm{I}_{3}$ were made with Colloid III, which had stood in the presence of ether and alcohol for several days. When the layers separated quickly, indicating considerable difference in specific gravity, sometimes a little colloid would be seen in the upper layer; in such cases the upper layer had a milky appearance 
TABLE IV-Distribution Data

Colloid I

\begin{tabular}{|c|c|c|c|c|c|c|c|}
\hline & \multirow[b]{2}{*}{ Ether } & \multicolumn{2}{|c|}{ Upper layer } & & \multicolumn{2}{|c|}{ Lower layer } & \multirow{2}{*}{$\begin{array}{c}\text { Ratio } \\
\begin{array}{c}\mathrm{Gms} . \mathrm{As}_{2} \mathrm{~S}_{8} \\
\text { in L. L. }\end{array} \\
\begin{array}{c}\mathrm{Gms} . \mathrm{As}_{2} \mathrm{~S}_{3} \\
\text { in U. L. }\end{array}\end{array}$} \\
\hline & & $\begin{array}{c}\mathrm{As}_{2} \mathrm{~S}_{3} \\
\text { per } \\
\text { liter } \\
\text { Gram }\end{array}$ & $\begin{array}{c}\mathrm{As}_{2} \mathrm{O}_{5} \\
\text { per } \\
\text { liter } \\
\text { Gram }\end{array}$ & & $\begin{array}{c}\mathrm{As}_{2} \mathrm{~S}_{3} \\
\text { per } \\
\text { liter } \\
\text { Grams }\end{array}$ & $\begin{array}{c}\mathrm{As}_{2} \mathrm{O}_{6} \\
\text { per } \\
\text { liter }\end{array}$ & \\
\hline ( I) & $0.55^{2}$ & 0.177 & 0.035 & - & $5.6 I$ & - & 3 I. 6 \\
\hline & 0.559 & 0.293 & 0.030 & - & 15.62 & O.IO4 & $53 \cdot 4$ \\
\hline \multicolumn{8}{|c|}{ COLLOID II } \\
\hline 3) & 0.536 & 0.875 & 0.046 & - & 4.86 & 0.046 & $5 \cdot 56$ \\
\hline 4) & 0.547 & 0.764 & 0.030 & - & $7 \cdot 32$ & 0.058 & $9 \cdot 5^{8}$ \\
\hline \multicolumn{8}{|c|}{ COLLOID III } \\
\hline (5) & 0.554 & 0.600 & 0.023 & - & 7.95 & 0.028 & 13.2 \\
\hline (6) & 0.555 & 0.556 & 0.023 & - & $7 \cdot 75$ & 0.046 & I 3.9 \\
\hline$(7)$ & 0.592 & $0.086 \mathrm{I}$ & $0.02 \mathrm{I}$ & - & 9.55 & - & I I I.O \\
\hline (8) & 0.571 & 0.0492 & $0.02 \mathrm{I}$ & - & 5.26 & 0.046 & 107.0 \\
\hline (9) & 0.537 & 0.613 & 0.046 & - & $5 \cdot 15$ & - & $8.4 \mathrm{I}$ \\
\hline (10) & 0.566 & 0.516 & 0.025 & - & 15.18 & - & 29.4 \\
\hline (I I) & 0.562 & 0.226 & 0.028 & 一 & 4.80 & - & $2 \mathrm{I} \cdot 2$ \\
\hline (I2) & $0.54 \mathrm{I}$ & 0.0674 & 0.023 & - & 2. 19 & - & $32 \cdot 5$ \\
\hline (13) & $0.54 \mathrm{I}$ & 0.0588 & 0.030 & - & I. 78 & - & 30.3 \\
\hline \multicolumn{8}{|c|}{ ColloId IV } \\
\hline (I4) & $0.55 \mathrm{I}$ & 0.669 & 0.013 & - & 10.0 & 0.030 & I 4.9 \\
\hline (15) & 0.544 & 2. 18 & 0.018 & - & I6. I & 0.069 & $7 \cdot 38$ \\
\hline (I6) & 0.545 & 0.976 & 0.014 & - & I I. 3 & 0.023 & I I . 6 \\
\hline (I 7) & 0.535 & 0.971 & o.OII & - & 6.62 & 0.021 & $6.8 \mathrm{I}$ \\
\hline (I8) & 0.544 & $0.2 \mathrm{I} 2$ & 0.046 & 一 & $5 \cdot 44$ & 0.122 & 25.6 \\
\hline \multicolumn{8}{|c|}{ Colloid V } \\
\hline & & & & $\begin{array}{l}\text { Time in } \\
\text { ice bath }\end{array}$ & & & \\
\hline (I9) & $0.5^{62}$ & 0.283 & 0.025 & 3 hrs. & 6.86 & 0.064 & 24.2 \\
\hline (20) & 0.566 & I. I I & 0.014 & 5 hrs. & 20.6 & 0.104 & 18.5 \\
\hline (2I) & 0.567 & 0.223 & 0.012 & 4 hrs. & 10.42 & O. I IO & 46.7 \\
\hline$(22)$ & 0.566 & $0.025^{8}$ & 0.025 & $7 \mathrm{hrs}$. & 5.29 & 0.122 & 205.0 \\
\hline
\end{tabular}

and was not clear as in the case of true distribution, Point 7 illustrates this condition. 
In the experiments with Colloid IV it was planned to have the upper layer much more concentrated with respect to the colloid than in former determinations. The points do not lie on a smooth curve.

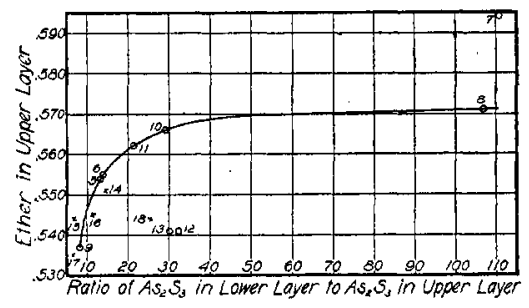

Fig. IV-Distribution plot: $\bigcirc$ colloid $3 ;+$ colloid $_{4}$

In Experiment I8, also with Colloid IV, the solutions were made up at the close of a day's work, and-to judge by the eye-the concentration of the colloid in the lower layer was about ten times as great as in the upper. The whole was then allowed to stand at room temperature over night, with the glass stopper firmly tied in to prevent loss of vapor. Next day, after cooling and shaking, it was at once apparent that the amount of colloid in the upper layer had greatly diminished; and on making the usual density determinations and analyses, a ratio was obtained which is much greater than fits in with the other experiments with the same colloid.

The measurements with Colloid V (19-22) were made with the object of obtaining phases of the same composition as regards ether, water and alcohol, but widely different in the concentration of the colloid. The method was to get the layers as nearly as possible of the right composition, and then to take the specific gravity of a portion of the upper layer, and, guessing at the correction for arsenious sulphide, obtain its composition from Fig. III. This portion was then replaced, a small amount of the proper liquid added in order to approach more nearly the desired density, and the whole was shaken and allowed to separate again. These operations were repeated until an upper layer of the desired density 
was obtained. Fair success was had in getting closely the same composition of phases as regards the organic liquids and water; but as seen in Table IV, the arsenic concentration ratios varied greatly.

\section{Conclusions and Summary}

Data have been obtained for the binodal curve and tielines in the system Alcohol-water-ether at $0^{\circ} \mathrm{C}$; the densities of the phases at equilibrium have been determined; and a method of allowing for the effect of colloidally dissolved arsenious sulphide on these densities has been checked experimentally. As regards alcohol, water and ether, the compositions of the phases at equilibrium are not affected by the presence of colloidal arsenious sulphide.

Colloidal solutions of arsenious sulphide containing alcohol and ether are more transparent and are less readily coagulated by salts than aqueous solutions, and there is not so much difference between the amounts of different salts needed to coagulate them.

Solutions containing alcohol, water, ether, and arsenious sulphide undergo change on standing; the amount of arsenic found in the filtrate after coagulation with acid increases, and the proportion of colloid going into the upper (more ethereal) layer on distribution decreases.

Quantitative measurements of the distribution of the arsenious sulphide between the two phases have been made; they show that when one and the same colloid preparation is used, and the measurements are carried out under the same conditions as to agitation, time of standing, etc., the ratio of distribution varies continuously with the composition of the phases; and that, other things being equal, the fraction of the arsenious sulphide going into the upper layer decreases with increase in the concentration of the sulphide.

My thanks are due to Prof. W. Lash Miller, under whose direction these experiments were carried out in the University of Toronto, during the winter of I9I I-IgI2. 